\title{
ROC curves in cost space
}

\author{
José Hernández-Orallo • Peter Flach • César Ferri
}

Received: 23 September 2012 / Accepted: 10 January 2013 / Published online: 23 February 2013

(C) The Author(s) 2013

\begin{abstract}
ROC curves and cost curves are two popular ways of visualising classifier performance, finding appropriate thresholds according to the operating condition, and deriving useful aggregated measures such as the area under the ROC curve $(A U C)$ or the area under the optimal cost curve. In this paper we present new findings and connections between ROC space and cost space. In particular, we show that ROC curves can be transferred to cost space by means of a very natural threshold choice method, which sets the decision threshold such that the proportion of positive predictions equals the operating condition. We call these new curves rate-driven curves, and we demonstrate that the expected loss as measured by the area under these curves is linearly related to $A U C$. We show that the rate-driven curves are the genuine equivalent of ROC curves in cost space, establishing a point-point rather than a point-line correspondence. Furthermore, a decomposition of the rate-driven curves is introduced which separates the loss due to the threshold choice method from the ranking loss (Kendall $\tau$ distance). We also derive the corresponding curve to the ROC convex hull in cost space; this curve is different from the lower envelope of the cost lines, as the latter assumes only optimal thresholds are chosen.
\end{abstract}

Keywords Cost curves $\cdot$ ROC curves $\cdot$ Cost-sensitive evaluation $\cdot$ Ranking performance $\cdot$ Operating condition · Kendall tau distance $\cdot$ Area Under the ROC Curve (AUC)

Editors: Hendrik Blockeel, Kristian Kersting, Siegfried Nijssen, and Filip Železný.

J. Hernández-Orallo · C. Ferri (凶)

Departament de Sistemes Informàtics i Computació, Universitat Politècnica de València, València,

Spain

e-mail: cferri@dsic.upv.es

J. Hernández-Orallo

e-mail: jorallo@dsic.upv.es

P. Flach

Department of Computer Science, University of Bristol, Bristol, UK

e-mail: peter.flach@bristol.ac.uk 


\section{Introduction and motivation}

ROC curves (Swets et al. 2000; Fawcett 2006) constitute a popular and highly useful graphical representation of classifier performance. A point on a ROC curve visualises the true and false positive rates achieved by a particular decision threshold. A monotonic curve is obtained by sweeping through all possible decision thresholds, and the area under the curve $(A U C)$ corresponds to the proportion of correctly ranked pairs of positive and negative examples. ROC curves can be used to identify optimal thresholds that yield points on a ROC curve's convex hull, as well as regions where one classifier dominates another. Operating conditions (class and misclassification cost distributions) manifest themselves as straight isometrics in ROC space.

Classification loss at a particular decision threshold is not visualised directly in ROC curves, but has to be inferred from the true and false positive rate and operating condition. Cost curves were proposed by Drummond and Holte $(2000,2006)$ as an alternative to ROC curves that explicitly visualise loss on the $y$-axis against the operating condition on the $x$ axis. For example, if we fix the decision threshold and the class distribution and vary the relative misclassification cost $c$ of one of the classes, then loss will vary linearly with $c$ and we obtain a cost line. Since a fixed threshold corresponds to a point in ROC space, this suggests a point-line duality between the two representations as noted by Drummond and Holte (2006) (see Fig. 1). Further correspondences include that between the ROC convex hull and the lower envelope of a classifier's cost lines, which both arise from optimal decision thresholds. Thus, cost curves allow us to not only identify regions of dominance, but quantify exactly the advantage in classification loss of the dominating classifier over the dominated one at a particular operating condition.

However, the correspondence between ROC space and cost space is incomplete to date. In particular, Drummond and Holte (2006) did not propose a cost space equivalent of a ROC curve. Furthermore, while linear interpolation between points in ROC space has a clear interpretation as a random choice between two decision thresholds, no similar construct has been proposed for cost space. In this paper we solve these and related open problems by deriving the exact equivalent of a ROC curve in cost space. The missing link here is a particular way of translating operating conditions into decision thresholds that is well-suited for models that are good rankers but do not necessarily produce well-calibrated scores. This rate-driven threshold choice method sets the decision threshold such that the proportion or rate of positive predictions equals the operating condition. This leads to a piecewise cost curve where each segment in a ROC curve corresponds to a quadratic cost curve segment. We show how this curve is the real equivalent in cost space to ROC curves. The area under this rate-driven curve can be easily shown to be linearly related to AUC. A decomposition of the rate-driven curve is also derived, leading to a new curve, which we call Kendall curve, because it depicts ranking performance (Kendall $\tau$ distance to the perfect ranker) in cost space. Thus, rather than an incomplete point-line duality as suggested by Drummond and Holte (2006), we show a complete point-to-point correspondence between ROC space and cost space for classifiers employing the rate-driven threshold choice method. Under this interpretation ROC curves and cost curves are truly two sides of the same coin.

The paper is organised as follows. Section 2 introduces basic notation and definitions. Section 3 introduces a new threshold choice method based on rates, which leads to the rate-driven curves, showing classification performance, and its area is shown to be a linear function of $A U C$, as shown in Sect. 4. Section 5 investigates how these curves can be decomposed, introducing a new curve of ranking performance called Kendall curve. Section 6 illustrates the point-point correspondence between ROC space and cost space. This 

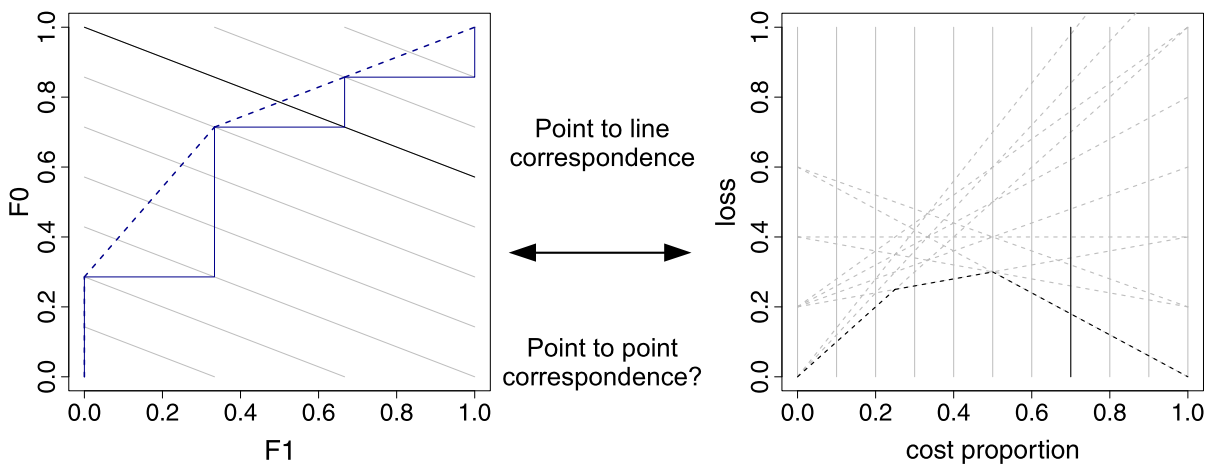

Fig. 1 Point-to-line correspondence between ROC curves and (optimal) cost curves (some of the terms used in this figure are defined in Sects. 2 and 3). Left: A ROC curve corresponding to the ranking 0010001010. The diagonal lines are rate isometrics (lines connecting points with the same predicted positive rate $r$; these have slope $-\pi_{1} / \pi_{0}$ and intercept $r / \pi_{0}$, where $\pi_{0}$ and $\pi_{1}$ are the class priors), one for each possible split point in the ranking. The isometric (bold line) going through the top left-hand corner has rate $\pi_{0}$. Other rates can be achieved in expectation by means of a random choice between the two bordering split points. Right: the corresponding optimal cost curve (shown for cost proportions instead of skews). Each of the 11 points on the ROC curve corresponds to one of the 11 cost lines (dashed). Furthermore, points on the ROC convex hull correspond to segments in the optimal cost curve. In particular, ROC point $(0 / 3,0 / 7)$ corresponds to the top cost line emanating from the origin (which only touches the optimal cost curve in the origin), ROC point $(0 / 3,2 / 7)$ corresponds to the ascending diagonal in cost space (which intersects with the first segment of the optimal cost curve), ROC point $(1 / 3,5 / 7)$ corresponds to the bottom cost line emanating from $(0,0.2)$ (which contributes the second segment of the optimal cost curve), point $(2 / 3,6 / 7)$-not strictly part of the convex hull since it connects two segments with the same slope-corresponds to the bottom cost line emanating from $(0,0.4)$ (which touches the optimal cost curve at cost $=0.5)$, and ROC point $(3 / 3,7 / 7)$ corresponds to the bottom cost line emanating from $(0,0.6)$ (which establishes the final segment of the cost curve). The vertical line in bold represents the rate $\pi_{0}=0.7$

applies also to the convex hull, whose equivalent curve in cost space, the convex skull, is discovered, and its relation with the lower envelope of the cost lines is analysed in Sect. 7. Section 8 shows how rate-driven cost curves and Kendall curves can be used in practice, especially focusing on screening applications and other classification settings where partial areas might be useful. Section 9 closes the paper with a discussion of the results.

\section{Notation and basic definitions}

In this section we introduce some basic notation and the notions of ROC curves, cost curves and the way expected loss is aggregated using a threshold choice method.

Examples or instances are taken from an instance space. The instance space is denoted $X$ and the output space $Y$. Elements in $X$ and $Y$ will be referred to as $x$ and $y$ respectively. For this paper we will assume binary classifiers, i.e., $Y=\{0,1\}$, where 0 is the positive class and 1 is the negative class. A crisp or categorical classifier is a function that maps examples to classes. A model or scoring classifier is a function $m: X \rightarrow \mathbb{R}$ that maps examples to scores on an unspecified scale, such that a higher score expresses a stronger belief that the example is negative. ${ }^{1}$ In order to make predictions in the $Y$ domain, a model can be converted to

\footnotetext{
${ }^{1}$ We use 0 for the positive class and 1 for the negative class, but scores increase with $\hat{p}(1 \mid x)$. That is, a ranking from strongest positive prediction to strongest negative prediction has non-decreasing scores. This is the same convention as used by, e.g., Hand (2009).
} 
a crisp classifier by fixing a decision threshold $t$ on the scores. Given a predicted score $s=m(x)$, the instance $x$ is classified in class 1 if $s>t$, and in class 0 otherwise.

For a given, unspecified model and population from which data are drawn, we denote the score density for class $k$ by $f_{k}$ and the cumulative distribution function by $F_{k}$. Thus, $F_{0}(t)=\int_{-\infty}^{t} f_{0}(s) d s=P(s \leq t \mid 0)$ is the proportion of class 0 points correctly classified if the decision threshold is $t$, which is the sensitivity or true positive rate at $t$. Similarly, $F_{1}(t)=\int_{-\infty}^{t} f_{1}(s) d s=P(s \leq t \mid 1)$ is the proportion of class 1 points incorrectly classified as 0 or the false positive rate at threshold $t ; 1-F_{1}(t)$ is the true negative rate or specificity. Given a data set $D \subset\langle X, Y\rangle$, we denote by $D_{k}$ the subset of examples in class $k \in\{0,1\}$, and set $\pi_{k}=\left|D_{k}\right| /|D|$. We will use the term class proportion for $\pi_{0}$ (other terms such as 'class ratio' or 'class prior' have been used in the literature). Given a model and a threshold $t$, we denote by $R(t)=\pi_{0} F_{0}(t)+\pi_{1} F_{1}(t)$ the predicted positive rate, i.e., the proportion of examples that will be predicted positive if the decision threshold is set at $t$.

\subsection{Operating conditions and overall loss}

When a classification model is applied, the conditions or context might be different to those used during its training. In fact, a model can be used in several contexts, with different results. A context can imply different class proportions, different cost over examples (either for the attributes, for the class or any other kind of cost), or some other details about the effects that the application of a model might entail and the severity of its errors.

One general approach to cost-sensitive learning assumes that the cost does not depend on the example but only on its class. In this way, misclassification costs are usually simplified by means of cost matrices, where we can express that some misclassification costs are higher than others (Elkan 2001). Typically, the costs of correct classifications are assumed to be 0 . This means that for binary classifiers we can describe the cost matrix by two values $c_{k} \geq 0$, representing the misclassification cost of an example of class $k$. We can normalise the costs by setting $b=c_{0}+c_{1}$ and $c=c_{0} / b$; we will refer to $c$ as the cost proportion. We set $b=2$ so that loss is commensurate with error rate (which assumes $c_{0}=c_{1}=1$ ).

The loss which is produced at a decision threshold $t$ and a cost proportion $c$ is then given by the formula:

$$
\begin{aligned}
Q_{\text {cost }}(t ; c) & \triangleq c_{0} \pi_{0}\left(1-F_{0}(t)\right)+c_{1} \pi_{1} F_{1}(t) \\
& =2\left\{c \pi_{0}\left(1-F_{0}(t)\right)+(1-c) \pi_{1} F_{1}(t)\right\}
\end{aligned}
$$

We often are interested in analysing the influence of class proportion and cost proportion at the same time. Since the relevance of $c_{0}$ increases with $\pi_{0}$, an appropriate way to consider both at the same time is by the definition of skew, which is a normalisation of their product:

$$
z \triangleq \frac{c_{0} \pi_{0}}{c_{0} \pi_{0}+c_{1} \pi_{1}}=\frac{c \pi_{0}}{c \pi_{0}+(1-c)\left(1-\pi_{0}\right)}
$$

From Eq. (1) we obtain

$$
\frac{Q_{\text {cost }}(t ; c)}{c_{0} \pi_{0}+c_{1} \pi_{1}}=z\left(1-F_{0}(t)\right)+(1-z) F_{1}(t) \triangleq Q_{s k e w}(t ; z)
$$

We will assume that the operating condition is either defined by the cost proportion (using a fixed class distribution) or by the skew. 


\subsection{Threshold choice methods}

A key issue when applying a model to several operating conditions is how the threshold is chosen in each of them. If we work with a crisp classifier, this question vanishes, since the threshold is already settled. However, in the general case when we work with a model as a scoring or probabilistic classifier, we have to decide how to establish the threshold. The crucial idea is the notion of threshold choice method, a function $T(c)$ or $T(z)$ which converts an operating condition (cost proportion or skew) into an appropriate threshold for the model. There are several reasonable options for the function $T$ : we can set a fixed threshold for all operating conditions; we can set the threshold by looking at the ROC curve (or its convex hull) and using the cost proportion or the skew to intersect the ROC curve (as ROC analysis does); we can set a threshold looking at the estimated scores, especially when they represent probabilities; or we can set a threshold independently from the rank or the scores. For a comprehensive account of threshold choice methods, we refer to Hernández-Orallo et al. (2012). The way in which we set the threshold may dramatically affect performance.

In many real-world problems, when we have to evaluate or compare classification models, we do not know the cost proportion or skew that will apply during deployment time. One general approach is to evaluate the model on a range of possible operating points. From this interpretation, Adams and Hand (1999) suggest to set a distribution over the set of possible operating points and integrate over them. In this way, we can define the overall or average expected loss in a range of situations as follows:

$$
L_{c} \triangleq \int_{0}^{1} Q_{\text {cost }}\left(T_{\text {cost }}(c) ; c\right) w_{\text {cost }}(c) d c
$$

where $Q_{\text {cost }}(t)$ is the expected cost for threshold $t$ as defined in Eq. (1), $T_{\text {cost }}$ is a threshold choice method which maps cost proportions to thresholds, and $w_{\cos t}(c)$ is a distribution for costs in $[0,1]$. We can define a similar construction for skews instead of cost proportions:

$$
L_{z} \triangleq \int_{0}^{1} Q_{\text {skew }}\left(T_{\text {skew }}(z) ; z\right) w_{\text {skew }}(z) d z
$$

In the rest of the paper we will assume the uniform distribution for $w_{\text {cost }}$ and $w_{\text {skew }}$, using $U(c)$ and $U(z)$ as subscripts.

\subsection{ROC curves and cost curves}

The ROC curve (Swets et al. 2000; Fawcett 2006) is defined as a plot of $F_{1}(t)$ (i.e., false positive rate at decision threshold $t$ ) on the $x$-axis against $F_{0}(t)$ (true positive rate at $t$ ) on the $y$-axis, with both quantities monotonically non-decreasing with increasing $t$ (remember that scores increase with $\hat{p}(1 \mid x)$ and 1 stands for the negative class). The area under the ROC curve is denoted by $A U C$. $A O C=1-A U C$ denotes the area above the ROC curve. Figure 1 (left) shows a ROC curve with $A U C=13 / 21$ and $A O C=8 / 21$. This model will be a running example for the rest of the paper. An important concept in ROC analysis is the notion of ROC isometrics (Flach 2003). A ROC isometric is a line (or curve) that represents the points with the same value for a given measure. If we focus on loss isometrics, we have that they only depend on the skew $z$, leading to straight lines (called iso-cost lines) whose slope equals $\frac{1-z}{z}$. Consequently, given a skew, we just slide a straight line with the corresponding slope from the top-left corner $(0,1)$ until we touch the ROC curve. This point 
Fig. 2 The cost space of the model in Fig. 1 (again, we use cost proportions $c$ instead of skews $z$ ). The dashed lines represent all possible cost lines. The two points shown as (big) dots correspond to two possible crisp classifiers evaluated with cost values $c=0.4$ and $c=0.6$ respectively. The point at $c=0.4$ is the result given by a classifier with $F_{1}=1 / 3$ and $F_{0}=5 / 7$, leading to a cost line from 0.2 to 0.4 . This gives a cost of 0.28 for $c=0.4$. The point at $c=0.6$ is the result given by a classifier with $F_{1}=1 / 3$ and $F_{0}=4 / 7$, leading to a cost line from 0.2 to 0.6 . This gives a cost of 0.44 for $c=0.6$

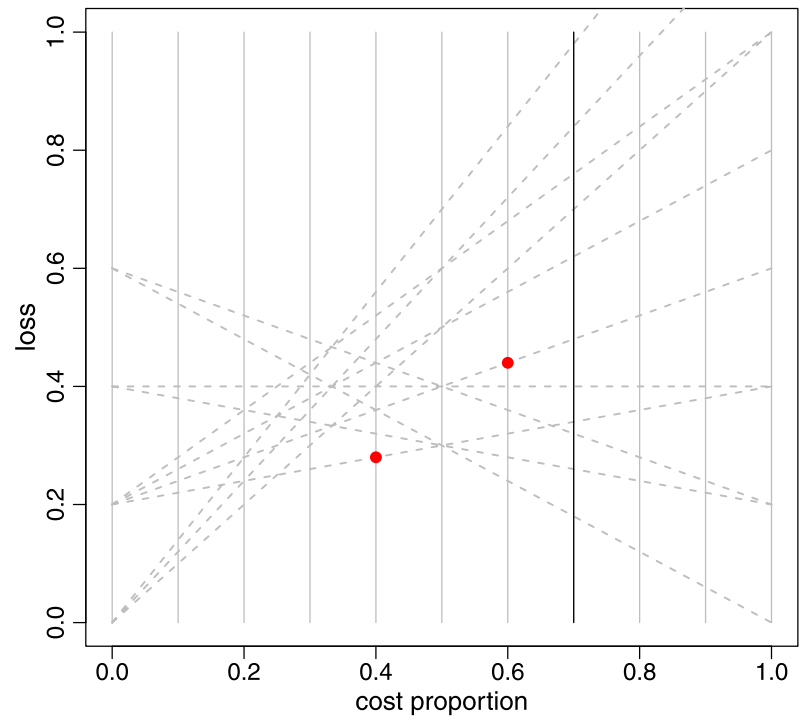

gives the optimal threshold for that skew and leads to optimal decisions in case the ROC curve reliably represents the behaviour of the classifier for the data at hand.

Cost space, as defined by Drummond and Holte (2006) has $Q_{\text {skew }}(t ; z)$ on the $y$-axis against skew $z$ on the $x$-axis (Drummond and Holte use the term 'probability cost' rather than skew). We can plot cost space for cost proportions $c$ instead of skews on the $x$-axis, as shown in Fig. 2. In cost space, loss isometrics are horizontal lines. This simplifies the procedure of determining the loss resulting from a given cost proportion or skew. In particular, finding the classifier that minimises the loss for a given skew on the $x$-axis amounts to finding the lowest cost line or cost curve at that $x$-value.

While ROC curves arise from varying the classifier's thresholds (interpolating between the resulting points in the empirical case), curves in cost space are established by considering a range of skews or cost proportions. So a cost curve as a function of $z$ in our notation is: $C C_{\text {skew }}(z) \triangleq Q_{\text {skew }}(T(z) ; z)=z\left(1-F_{0}(T(z))\right)+(1-z) F_{1}(T(z))$, and similarly for cost proportions using $Q_{\text {cost }}$.

The threshold choice method $T$ is what characterises the cost curve. If we choose a function $T$ which sets a fixed threshold $t$ regardless of the operating condition, then we have that the loss varies linearly in cost space. For the interval of thresholds $t$ that give the same class assignments we clearly have the same line, which is called the cost line (not to be confused with loss isometrics in ROC analysis). A cost line visualises how loss at a fixed threshold $t$ changes between $F_{1}(t)$ for $z=0$ and $1-F_{0}(t)$ for $z=1$, when using skews. Using cost proportions the cost lines run from $2 \pi_{1} F_{1}(t)$ for $c=0$ to $2 \pi_{0}\left(1-F_{0}(t)\right)$ for $c=1$. This is illustrated in Fig. 2. From all cost lines we can choose line segments (depending on where we change the threshold) and by piecewise connecting them we have a 'hybrid cost curve' (Drummond and Holte 2006).

One way of choosing these segments is by considering the optimal threshold, which is defined as follows:

$$
T_{\text {skew }}^{o}(z) \triangleq \underset{t}{\arg \min }\left\{Q_{\text {skew }}(t ; z)\right\} .
$$


The optimal or minimum cost curve is then the lower envelope of all the cost lines. The cost curve for this optimal choice is defined as $C C_{\text {skew }}^{o}(z) \triangleq Q_{\text {skew }}\left(T_{\text {skew }}^{o}(z) ; z\right)$. Similar expressions are obtained for cost proportions. Figure 1 (right) shows the optimal cost curve (using cost proportions) for the running example.

Note that our notation makes it explicit that other curves can be obtained in cost space by changing the threshold choice method $T$.

\section{The rate-driven threshold choice method}

In Sect. 2.2 we mentioned that there are several ways to choose a threshold given a soft or probabilistic classifier. One of the differences between ROC curves and cost curves is precisely that the former is independent of the threshold choice method, while the cost curve completely depends on this choice. As mentioned above, classical cost curves represent how the loss of a classifier changes with the operating condition assuming the optimal threshold choice method. However, there is in general no guarantee that we will be able to find the optimal threshold choice at deployment time. Furthermore, on many occasions, even assuming that the optimal choice on the plot could ultimately match the optimal choice in the deployment data, we have to consider that ROC analysis and cost curves are not always used, and decisions may be made by choosing the threshold in a different way.

An alternative option is the score-driven threshold choice method, which assumes a probabilistic classifier outputting scores between 0 and 1 and just sets $T(c)=c$. An assumption of equal misclassification costs might thus justify a threshold of 0.5 on naive Bayes' estimates of the posterior probability. If the probability estimates are well-calibrated this is a reasonable choice from the point of view of risk minimisation. The score-driven threshold choice method leads to a different curve in cost space, which has been termed the Brier curve (Hernández-Orallo et al. 2011) since its area equals the Brier score, a very common metric for evaluating probabilistic classifiers. This threshold choice method is particularly sensitive to how the probabilities are estimated. If estimated probabilities are highly concentrated (e.g., if half of them are in the range $[0.4,0.6]$ ) and we use a probability in this range as a threshold (e.g., 0.55), a minor variation in the estimated probabilities will change predictions and hence loss dramatically. This problem also affects the optimal threshold choice method, because we may determine the optimal threshold on a ROC curve plotted with a validation data set and then take the score (or estimated probability) that leads to this optimal choice. Clearly, the score-driven threshold choice method and the optimal threshold choice method are equivalent when the model is perfectly calibrated.

A third way of determining a decision threshold is by considering the proportion of positives that we want to predict. If we find a point on the ROC curve (plotted with a training or validation data set) that we want to use to set the threshold, we can just calculate the predicted positive rate (the proportion of positive predictions) and use this rate as the reference for the deployment data set. The only limitation of using rates instead of a numerical score is that rates only make sense when we have a batch of predictions. Nonetheless, this is a very common situation. This idea of making decisions based on the rate instead of the scores leads to the rate-driven threshold choice method below.

Recall that the predicted positive rate, abbreviated to rate, is defined as $R(t)=\pi_{0} F_{0}(t)+$ $\pi_{1} F_{1}(t)$. For skews we have $R_{z}(t)=\left(F_{0}(t)+F_{1}(t)\right) / 2$. The following threshold choice method sets the threshold to achieve a rate equal to the operating condition. 
Definition 1 The rate-driven threshold choice method for cost proportions is defined as

$$
T_{\text {cost }}^{r d}(c) \triangleq R^{-1}(c)
$$

Similarly, for skews:

$$
T_{\text {skew }}^{r d}(z) \triangleq R_{z}^{-1}(z)
$$

We can achieve any rate, provided $F_{0}$ and $F_{1}$ are continuous. In the empirical case this can be achieved by interpolation, as is customary in ROC curves. Thus, to achieve a rate that is between two split points of a ranking, we randomly choose between the split points in such a way that the desired rate is achieved in expectation. Figure 1 (left) illustrates this graphically.

Example 1 Following the running example in Fig. 1, and assuming that we have scores $\{-3.20,-2.13,-1.15,-0.18,0.21,0.45,1.47,1.49,1.93,4.72\}$ we can explain how this threshold choice method works to make decisions, especially in the empirical case. If we are given, e.g., a cost proportion of $c=0.725$, and we only have ten examples in our data set, the rate 0.725 cannot be achieved with a single split point. So, the rate which corresponds to cost proportion 0.725 must be achieved in expectation by stochastic interpolation between the closest rate isometrics. In this case, we have isometric $A$ with rate 0.7 (making 7 positive predictions out of 10) with any threshold $1.47 \leq t<1.49$, and isometric $B$ with rate 0.8 (making 8 positive predictions) with any threshold $1.49 \leq t<1.93$. We stochastically choose between these by tossing a biased coin with probability $0.75=(0.8-0.725) /(0.8-0.7)$ of choosing $A$. Note that this is quite different to choosing just the closest rate isometric, which in this case would be to choose 0.7 as the rate, leading to a (somewhat simpler but) biased decision rule. In any case, we see that the magnitudes of the scores are irrelevant for the rate-driven threshold choice method. Only the ranks of the scores matter.

Under this threshold choice method the loss at threshold $t=T_{\text {cost }}^{r d}(c)$ and cost proportion $c$ can be entirely expressed in terms of $c$ :

$$
\begin{aligned}
Q_{\text {cost }}(t ; c) & \triangleq 2\left\{c \pi_{0}\left(1-F_{0}(t)\right)+(1-c) \pi_{1} F_{1}(t)\right\} \\
& =2\left\{c \pi_{0}+\pi_{1} F_{1}(t)-c\left[\pi_{0} F_{0}(t)+\pi_{1} F_{1}(t)\right]\right\} \\
& =2\left\{c\left(\pi_{0}-R(t)\right)+\pi_{1} F_{1}(t)\right\} \\
& =2\left\{c\left(\pi_{0}-c\right)+\pi_{1} F_{1}\left(R^{-1}(c)\right)\right\}
\end{aligned}
$$

In the last step we have used $t=R^{-1}(c)$ and so $c=R(t)$. The notation $F_{1}\left(R^{-1}(c)\right)$ stands for 'the false positive rate at the decision threshold which achieves rate $c$ ', usually achieved by interpolation between two classifiers.

It will be useful to derive an alternative expression for $Q_{\text {cost }}$ in terms of $F_{0}$ rather than $F_{1}$ :

$$
\begin{aligned}
Q_{\text {cost }}(t ; c) & \triangleq 2\left\{c \pi_{0}\left(1-F_{0}(t)\right)+(1-c) \pi_{1} F_{1}(t)\right\} \\
& =2\left\{c \pi_{0}-\pi_{0} F_{0}(t)+(1-c)\left[\pi_{0} F_{0}(t)+\pi_{1} F_{1}(t)\right]\right\} \\
& =2\left\{(1-c)\left(R(t)-\pi_{0}\right)+\pi_{0}\left(1-F_{0}(t)\right)\right\} \\
& =2\left\{(1-c)\left(c-\pi_{0}\right)+\pi_{0}\left(1-F_{0}\left(R^{-1}(c)\right)\right)\right\}
\end{aligned}
$$


where $F_{0}\left(R^{-1}(c)\right)$ means 'the true positive rate at the decision threshold which achieves rate $c^{\prime}$.

The rate-driven threshold choice method is a natural way of choosing the thresholds, especially when we only have a ranking or a poorly calibrated probabilistic classifier. While in this paper we use this method to make the connection between ROC space and cost space, it is a credible threshold choice method in itself, as an alternative to other methods. Clearly there are pros and cons for each threshold choice method. In particular, it is worth pointing out that the optimal threshold choice method utilises a ROC curve (and hence labelled data) to translate an operating condition into a threshold, unlike the score-driven and rate-driven methods. The ability to utilise this extra information provides the main appeal of the optimal threshold choice method, but also introduces the danger of overfitting if the ROC curve on which the optimal thresholds are determined is not representative. There is no guarantee that the optimal thresholds on the training or validation data are also optimal in the deployment context. Since in this paper the analysis concentrates on the case that the true probability distributions are known, this drawback of the optimal method may not always be apparent. Conversely, the score-driven and rate-driven methods can be expected to be more robust against overfitting the decision threshold. The connection between these threshold choice methods has been thoroughly explored in Hernández-Orallo et al. (2012), by comparing the aggregated cost for all possible cost proportions.

However, the definition of a curve from the rate-driven threshold choice method (including the interpolation of points between rates) and the analysis of the exact meaning of each point (and each region) of the curve is yet to be explored. This is the aim of this paper.

\section{The rate-driven cost curve}

We now introduce a new kind of cost curve that allows us to establish a one-to-one correspondence between cost space and ROC space.

Definition 2 The rate-driven cost curve is defined as a plot of $Q_{\text {cost }}\left(T_{\text {cost }}^{r d}(c) ; c\right)=2\left\{c\left(\pi_{0}-\right.\right.$ $\left.c)+\pi_{1} F_{1}\left(R^{-1}(c)\right)\right\}$ on the $y$-axis against $c$ on the $x$-axis. We can analogously define a version for skews as $Q_{\text {skew }}\left(T_{\text {skew }}^{r d}(z) ; z\right)=z(1-2 z)+F_{1}\left(R_{z}^{-1}(z)\right)$ against $z$.

Figure 3 shows the rate-driven cost curve corresponding to the model in Fig. 1.

Note that the rate-driven cost curve is continuous if the ROC curve is; if the ROC curve is piecewise linear (e.g., because of linear interpolation in case of an empirical curve), the ratedriven cost curve is piecewise parabolic because of the quadratic $c$ term in $Q_{\text {cost }}$. Figure 3 demonstrates this.

We now show that the area under the rate-driven cost curve is related to the $A U C$. The expected rate-driven loss for a range of cost proportions is:

$$
L_{w_{\text {cost }}}^{r d} \triangleq \int_{0}^{1} Q_{\text {cost }}\left(T_{\text {cost }}^{r d}(c) ; c\right) w_{\text {cost }}(c) d c
$$

If we use the uniform distribution for $w_{\text {cost }}(c)$ the expected loss is equal to the area under the rate-driven cost curve.

Theorem 1 (Hernández-Orallo et al. 2012) Expected loss for uniform cost proportions using the rate-driven threshold choice method is linearly related to AUC as follows:

$$
L_{U}^{r d}=\pi_{1} \pi_{0}(1-2 A U C)+1 / 3
$$


Fig. 3 The rate-driven cost curve corresponding to the model in Fig. 1. The $x$-axis shows cost proportion. The plot also shows the rate isometrics in cost space, as vertical lines

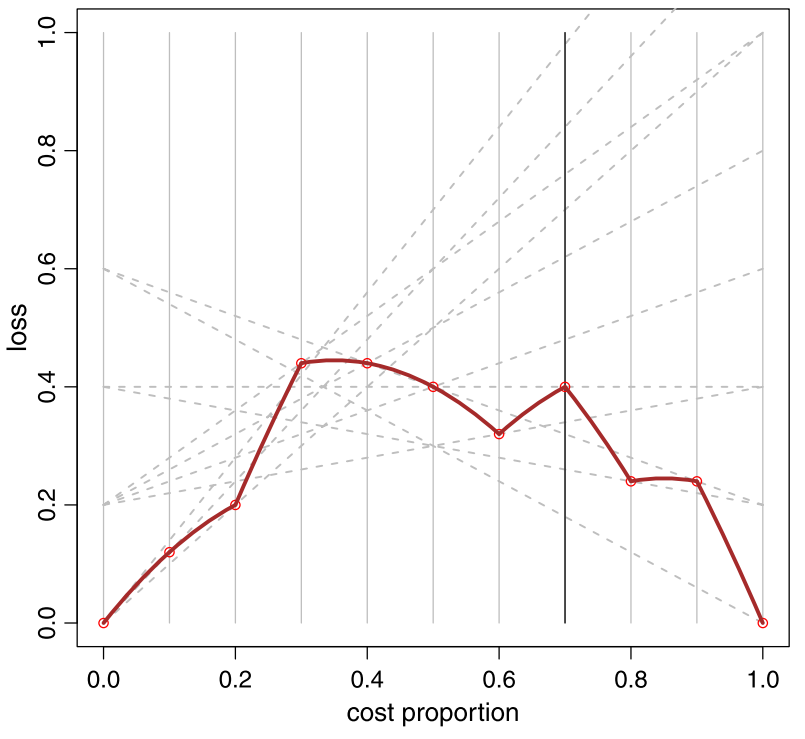

\section{Proof}

$$
\begin{aligned}
L_{U}^{r d} & \triangleq \int_{0}^{1} Q_{\text {cost }}\left(T_{\text {cost }}^{r d}(c) ; c\right) U(c) d c \\
& =\int_{0}^{1} 2\left\{c\left(\pi_{0}-c\right)+\pi_{1} F_{1}\left(R^{-1}(c)\right)\right\} d c \\
& =\int_{0}^{1} 2 c\left(\pi_{0}-c\right) d c+\int_{0}^{1} 2 \pi_{1} F_{1}\left(R^{-1}(c)\right) d c
\end{aligned}
$$

The first integral evaluates to $\left[c^{2} \pi_{0}-\frac{2 c^{3}}{3}\right]_{0}^{1}=\pi_{0}-2 / 3$. By a change of variable we have $c=R(t)$ and $d c=R^{\prime}(t) d t$ :

$$
\begin{aligned}
\int_{0}^{1} 2 \pi_{1} F_{1}\left(R^{-1}(c)\right) d c & =\int_{-\infty}^{+\infty} 2 \pi_{1} F_{1}(t) R^{\prime}(t) d t \\
& =2 \pi_{1} \int_{-\infty}^{\infty} F_{1}(t)\left\{\pi_{0} f_{0}(t)+\pi_{1} f_{1}(t)\right\} d t \\
& =2 \pi_{0} \pi_{1} \int_{-\infty}^{\infty} F_{1}(t) f_{0}(t) d t+2 \pi_{1}^{2} \int_{-\infty}^{\infty} F_{1}(t) f_{1}(t) d t \\
& =2 \pi_{0} \pi_{1}(1-A U C)+2 \pi_{1}^{2} \int_{0}^{1} F_{1}(t) d F_{1}(t) \\
& =2 \pi_{0} \pi_{1}(1-A U C)+\pi_{1}^{2}
\end{aligned}
$$

Summing both expressions and rearranging gives:

$$
\begin{aligned}
L_{U}^{r d} & =\pi_{0}-2 / 3+2 \pi_{0} \pi_{1}(1-A U C)+\pi_{1}^{2} \\
& =2 \pi_{1} \pi_{0}(1-A U C)+\pi_{1}\left(1-\pi_{0}\right)+\pi_{0}-2 / 3
\end{aligned}
$$



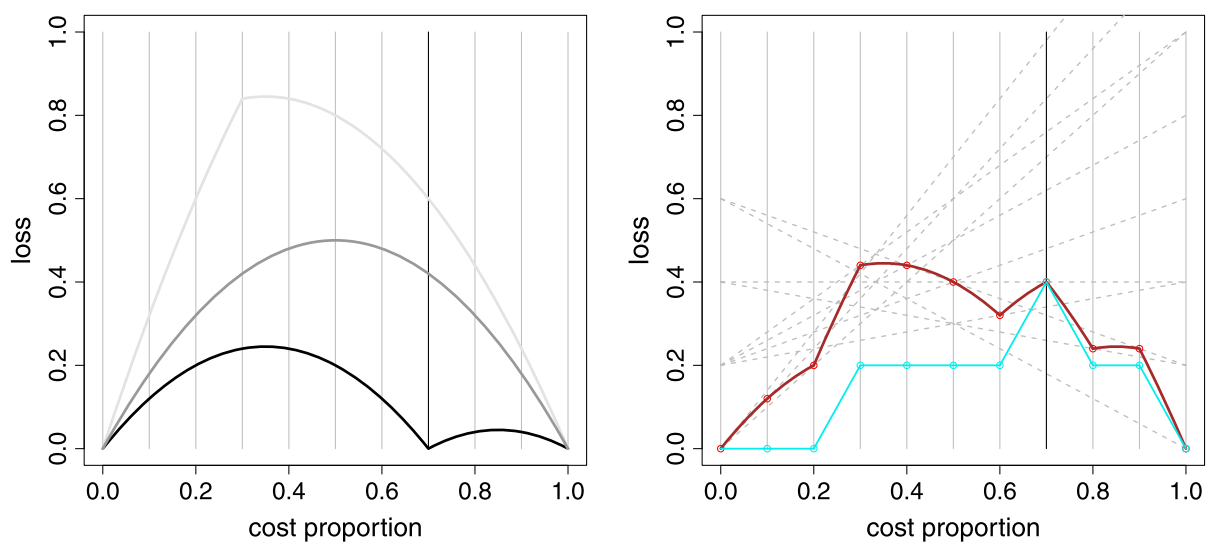

Fig. 4 Left: The worst rate-driven curve at the top, a classifier assigning the same score to all instances in the middle, and the optimal rate-driven curve at the bottom $\left(\pi_{0}\right.$ and $\pi_{1}$ as in the running example). Right: The Kendall curve for the running example (bottom) after subtracting the optimal rate-drive curve (left plot) from the rate-driven cost curve (top)

$$
=\pi_{1} \pi_{0}(1-2 A U C)+1 / 3
$$

Corollary 1 Expected rate-driven loss for uniform skews is $L_{U}^{r d}=(1-2 A U C) / 4+1 / 3$.

So the expected rate-driven loss for a random ranker is $1 / 3$. This reflects the fact that the threshold choice method takes advantage of knowing $c$ or $z$ : this lifts classification performance above that of a random classifier. On the other hand, the expected loss for a perfect ranker is non-zero (actually 1/12), because rate-driven thresholds are not always optimal. As we discussed in Sect. 3, this 'non-optimality' is the price we pay for using a method that is less prone to overfitting the decision threshold.

To illustrate, Fig. 4 (left) shows the rate-driven curve for the worst ranker possible (top), a ranker where all scores tie (middle) and a perfect ranker (bottom). We see that for the perfect ranker the rate-driven threshold choice method makes optimal choices for $c=0, c=$ $\pi_{0}=0.7$ and $c=1$ but sub-optimal choices for other operating conditions, which explains the non-zero area under the rate-driven curve. The reason why we can only have 0 loss at $c=\pi_{0}$ (apart from the two extremes) is because this is the only point where the predicted proportion of positives (the rate) matches the actual proportion of positives $\left(\pi_{0}\right)$. So, the ratedriven curve for a perfect ranker using the rate-driven threshold choice method can only be optimal in these three points. In order to be optimal for other points the only possibility is to change the threshold choice method.

\section{Decomposing the expected rate-driven loss: Kendall curves}

We note that the terms $2 c\left(\pi_{0}-c\right)$ in Eq. (9) and $2(1-c)\left(c-\pi_{0}\right)$ in Eq. (10) can be positive as well as negative. Combining their positive parts results in the rate-driven cost curve of a perfect ranker. An example of this curve was shown in Fig. 4 (left, bottom curve). 
Lemma 1 The rate-driven cost curve for a perfect ranker is defined as follows: ${ }^{2}$

$$
Q_{\text {cost }}^{*}\left(T_{\text {cost }}^{r d}(c) ; c\right)= \begin{cases}2 c\left(\pi_{0}-c\right) & \text { if } c \leq \pi_{0} \\ 2(1-c)\left(c-\pi_{0}\right) & \text { if } c \geq \pi_{0}\end{cases}
$$

with area $1 / 3-\pi_{0} \pi_{1}$

Proof The threshold where a perfect ranker achieves perfect classification is $R^{-1}\left(\pi_{0}\right)$, i.e. the left upper-hand corner in the ROC space. It follows that $F_{1}=0$ for $c \leq \pi_{0}$ and $F_{0}=1$ for $c \geq \pi_{0}$. We obtain the final expression if we consider $F_{1}=0$ for $c \leq \pi_{0}$ in Eq. (9) and we consider $F_{0}=1$ for $c \geq \pi_{0}$ in Eq. (10). The area comes from Theorem 1 with $A U C=1$.

Subtracting the expected rate-driven loss of a perfect ranker, which is the expected loss due to the rate-driven threshold choice method choosing non-optimal thresholds, from the expected loss given by Theorem 1 gives $2 \pi_{0} \pi_{1}(1-A U C)=2 \pi_{0} \pi_{1} A O C$ : this is the expected classification loss attributable to the model's ranking performance. Since the decomposition is pointwise for each $c$, we can construct a curve whose area can also be interpreted as the expected classification loss due to ranking performance. We call this new curve a Kendall curve, because, as we will see, its area is related to the Kendall $\tau$ distance (Kendall 1938) to the perfect ranking:

Definition 3 The Kendall curve is defined as follows:

$$
Q^{\tau}(c)= \begin{cases}Q_{0}^{\tau}(c)=2 \pi_{1} F_{1}\left(R^{-1}(c)\right) & \text { if } c \leq \pi_{0} \\ Q_{1}^{\tau}(c)=2 \pi_{0}\left(1-F_{0}\left(R^{-1}(c)\right)\right) & \text { if } c \geq \pi_{0}\end{cases}
$$

The Kendall curve shows, for each cost proportion $c$, the expected loss of the model, once the loss of a perfect ranker is discounted. This second loss is shared by all models.

Theorem 2 Any rate-driven cost curve can be decomposed into the rate-driven cost curve of a perfect ranker and the Kendall curve:

$$
Q_{\text {cost }}\left(T_{\text {cost }}^{r d}(c) ; c\right)=Q_{\text {cost }}^{*}\left(T_{\text {cost }}^{r d}(c) ; c\right)+Q^{\tau}(c)
$$

The area under the Kendall curve is $2 \pi_{0} \pi_{1} A O C$.

Proof This follows from Lemma 1 and Eqs. (9) and (10). The area is obtained from Theorem 1 and Lemma 1.

It is important to stress that the Kendall curve is the difference between two cost curves $\left(Q^{\tau}=Q_{\text {cost }}-Q_{\text {cost }}^{*}\right)$ but not itself a cost curve: notably, it does not intersect with cost lines as the rate-driven cost curves do. In other words, we can distinguish between the loss shared by all models_-since it originates from the rate-driven threshold choice method-and the loss originating from the model itself (expected classification loss attributable to the model's ranking performance). Figure 4 (right) shows a Kendall curve (bottom). If we focus on this

\footnotetext{
${ }^{2}$ Note that both conditions overlap for $c=\pi_{0}$, but this does not lead to ambiguity since both expressions are equal for $c=\pi_{0}$. 
curve, we see that some segments are horizontal and some others are diagonal. It is very

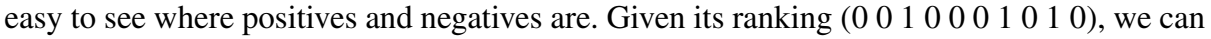
match this ranking to the curve (from left to right), and see that 0 s are shown horizontally and $1 \mathrm{~s}$ are shown diagonally, until the rate $\pi_{0}$ is reached ( 0.7 in the figure), where things swap, and $1 \mathrm{~s}$ are shown horizontally and 0 s are shown diagonally. Since the perfect ranking would be $\left(\begin{array}{lllllllll}0 & 0 & 0 & 0 & 0 & 0 & 0 & 1 & 1\end{array}\right)$, the Kendall curve shows how many discordant pairs will need to be swapped to get the perfect ranking ( 8 in total). This is precisely the Kendall $\tau$ distance to the perfect ranking, denoted by $K_{\tau}$. It is then easy to see that $K_{\tau}=\pi_{0} n \pi_{1} n A O C$, of which the area under the Kendall curve is just a normalisation with a factor $2 / n^{2}$. This relation between the Kendall $\tau$ distance and $A U C$ is not new. However, Kendall curves show this in a much more explicit way.

\section{Pointwise equivalence between ROC space and cost space}

The construction of the rate-driven curves and the derivation of the Kendall curves suggests that the correspondence between ROC space and cost space is much more direct than previously thought. The geometrical connection is given by the distance between the ROC curve and the ROC space square on one hand, and the height of the points in the rate-driven curve on the other hand. In what follows, we will work with empirical distributions. We will focus on the rate isometrics, which for the rate-driven threshold choice method are given by the costs which match a rate, i.e., $c=i / n$ for $i=0 \ldots n$.

We return to ROC curves to see that the area under the ROC curve can be obtained in a diagonal way and not horizontally, as the following two propositions show.

Proposition 1 Given a point in ROC space $\left(F_{1}\left(R^{-1}(c)\right), F_{0}\left(R^{-1}(c)\right)\right)$, the segment of the rate isometric connecting this point with the corresponding point of a perfect classifier has length:

$$
\begin{aligned}
& D_{0}(c) \triangleq \frac{F_{1}\left(R^{-1}(c)\right)}{\pi_{0}} \sqrt{\pi_{0}^{2}+\pi_{1}^{2}} \quad \text { if } c \leq \pi_{0} \\
& D_{1}(c) \triangleq \frac{\left(1-F_{0}\left(R^{-1}(c)\right)\right)}{\pi_{1}} \sqrt{\pi_{0}^{2}+\pi_{1}^{2}} \quad \text { if } c \geq \pi_{0}
\end{aligned}
$$

Proof If $c \leq \pi_{0}$, the perfect classifier goes with $f p r=0$ and increasing $t p r$ reaching the ROC heaven point $(0,1)$ for $c=\pi_{0}$. So, the length of the diagonal can be calculated as follows, using the definition of the rate $\left(R(t)=\pi_{0} F_{0}(t)+\pi_{1} F_{1}(t)\right.$, which, for the ratedriven threshold choice method leads to $\left.c=R(t)=\pi_{0} F_{0}\left(R^{-1}(c)\right)+\pi_{1} F_{1}\left(R^{-1}(c)\right)\right)$ :

$$
\begin{aligned}
D_{0}(c) & \triangleq d\left(\left(F_{1}\left(R^{-1}(c)\right), F_{0}\left(R^{-1}(c)\right)\right),\left(0, c / \pi_{0}\right)\right) \\
& =\sqrt{F_{1}\left(R^{-1}(c)\right)^{2}+\left(\frac{c}{\pi_{0}}-F_{0}\left(R^{-1}(c)\right)\right)^{2}} \\
& =\sqrt{F_{1}\left(R^{-1}(c)\right)^{2}+\left(\frac{\pi_{0}}{\pi_{0}} F_{0}\left(R^{-1}(c)\right)+\frac{\pi_{1}}{\pi_{0}} F_{1}\left(R^{-1}(c)\right)-F_{0}\left(R^{-1}(c)\right)\right)^{2}} \\
& =\sqrt{\left(1+\left(\frac{\pi_{1}}{\pi_{0}}\right)^{2}\right) F_{1}\left(R^{-1}(c)\right)^{2}}=\frac{F_{1}\left(R^{-1}(c)\right)}{\pi_{0}} \sqrt{\pi_{0}^{2}+\pi_{1}^{2}}
\end{aligned}
$$


If $c \geq \pi_{0}$, the perfect classifier goes from the heaven point $(0,1)$ to $(1,1)$ with $F_{0}\left(R^{-1}(c)\right)=$ 1 constant and increasing $F_{1}\left(R^{-1}(c)\right)$, from $c=\pi_{0}$ to $c=1$. So, the length of the diagonal can be calculated as follows:

$$
\begin{aligned}
D_{1}(c) & \triangleq d\left(\left(F_{1}\left(R^{-1}(c)\right), F_{0}\left(R^{-1}(c)\right)\right),\left(\frac{c-\pi_{0}}{\pi_{1}}, 1\right)\right) \\
& =\sqrt{\left(F_{1}\left(R^{-1}(c)\right)-\frac{c-\pi_{0}}{\pi_{1}}\right)^{2}+\left(1-F_{0}\left(R^{-1}(c)\right)\right)^{2}} \\
& =\sqrt{\left(1+\left(\frac{\pi_{0}}{\pi_{1}}\right)^{2}\right)\left(1-F_{0}\left(R^{-1}(c)\right)\right)^{2}} \\
& =\frac{\left(1-F_{0}\left(R^{-1}(c)\right)\right)}{\pi_{1}} \sqrt{\pi_{0}^{2}+\pi_{1}^{2}}
\end{aligned}
$$

Note that when $c=\pi_{0}$ then $D_{0}(c)=D_{1}(c)$.

Figure 1 shows a ROC curve and the rate isometrics. Proposition 1 calculates the length of the segment of each of these lines from the enclosing square (perfect classifier) to the actual ROC curve. The $A O C=1-A U C$ can be calculated from these diagonal segments as follows.

Proposition 2 Given a model with $n$ examples,

$$
A O C=\frac{\sum_{i=0}^{n \pi_{0}} D_{0}(i / n)+\sum_{i=n \pi_{0}+1}^{n} D_{1}(i / n)}{n \sqrt{\pi_{0}^{2}+\pi_{1}^{2}}}
$$

Proof The proof can be constructed geometrically by summing diagonal "units". It is easy to see geometrically that it is tantamount to sum diagonal "unit" segments than squares, as follows:

$$
A O C=\frac{\sum_{i=0}^{n \pi_{0}} F P\left(\frac{i}{n}\right)+\sum_{i=n \pi_{0}+1}^{n} n \pi_{0}-T P\left(\frac{i}{n}\right)}{\pi_{1} \pi_{0} n^{2}}
$$

Given that $F_{1}\left(R^{-1}(c)\right)=\frac{F P(c)}{n \pi_{1}}$ we obtain, from Proposition 1, that, for $c \leq \pi_{0}$ :

$$
F P(c)=n \pi_{1} F_{1}\left(R^{-1}(c)\right)=\frac{n \pi_{1} \pi_{0}}{\sqrt{\pi_{0}^{2}+\pi_{1}^{2}}} D_{0}(c)
$$

Similarly, with $F_{0}\left(R^{-1}(c)\right)=\frac{T P(c)}{n \pi_{0}}$, we have, for $c \geq \pi_{0}$ :

$$
n \pi_{0}-T P(c)=n \pi_{0}\left(1-F_{0}\left(R^{-1}(c)\right)\right)=\frac{n \pi_{1} \pi_{0}}{\sqrt{\pi_{0}^{2}+\pi_{1}^{2}}} D_{1}(c)
$$

Using these equivalences we get:

$$
A O C=\frac{\sum_{i=0}^{n \pi_{0}} \frac{n \pi_{1} \pi_{0}}{\sqrt{\pi_{0}^{2}+\pi_{1}^{2}}} D_{0}\left(\frac{i}{n}\right)+\sum_{i=n \pi_{0}+1}^{n} n \pi_{0}-\frac{n \pi_{1} \pi_{0}}{\sqrt{\pi_{0}^{2}+\pi_{1}^{2}}} D_{1}\left(\frac{i}{n}\right)}{\pi_{1} \pi_{0} n^{2}}
$$




$$
=\frac{\sum_{i=0}^{n \pi_{0}} D_{0}\left(\frac{i}{n}\right)+\sum_{i=n \pi_{0}+1}^{n} D_{1}\left(\frac{i}{n}\right)}{n \sqrt{\pi_{0}^{2}+\pi_{1}^{2}}}
$$

For the example in the left part of Fig. 6, we see that we have 8 squares above the curve, so $A O C$ is $8 /(7 \cdot 3)=8 / 21$. If we look at the diagonals, we have $0+1+1+2+1+1+1+$ $1+0+0+0=8$ unit segments, with $\sqrt{\pi_{0}^{2}+\pi_{1}^{2}} /\left(\pi_{1} \pi_{0} n\right)=0.762 /(0.3 \cdot 0.7 \cdot 10)=0.363$ length each. So the sum of the length of the diagonal isometrics from the ROC square to the ROC curve is $8 \cdot 0.363$. Dividing this value by $n \sqrt{\pi_{0}^{2}+\pi_{1}^{2}}=10 \cdot 0.762$ we get $8 / 21$.

Now we obtain a straightforward but important result which shows this exact correspondence between the two spaces (the length of the segments of the rate isometrics in ROC space and the loss value in cost space):

\section{Theorem 3}

$$
\begin{aligned}
& D_{0}(c)=\frac{Q_{0}^{\tau}(c)}{2 \pi_{1} \pi_{0}} \sqrt{\pi_{0}^{2}+\pi_{1}^{2}} \\
& D_{1}(c)=\frac{Q_{1}^{\tau}(c)}{2 \pi_{1} \pi_{0}} \sqrt{\pi_{0}^{2}+\pi_{1}^{2}}
\end{aligned}
$$

Proof From Proposition 1 and Theorem 2.

Corollary 2 AOC can be calculated by a summation over $Q^{\tau}$ :

$$
A O C=\frac{\sum_{i=0}^{n \pi_{0}} Q_{0}^{\tau}(i / n)+\sum_{i=n \pi_{0}+1}^{n} Q_{1}^{\tau}(i / n)}{2 \pi_{1} \pi_{0} n}=\frac{(2 / n) K_{\tau}}{2 \pi_{1} \pi_{0} n}
$$

Proof From Theorem 3 and Proposition 2.

This shows that $A O C$ can be computed efficiently and exactly by adding the heights of the points on the Kendall curve in cost space. The advantage of this calculation on cost space is that it connects this area to expected loss and it also provides a way to calculate 'partial' areas by considering particular cost ranges. Also, it can lead to different metrics by changing the $x$-axis to a different (non-uniform) distribution, if we are given (or we assume) some information about what costs proportions are more likely.

\section{Convex skull of the rate-driven curve}

One useful construction over ROC curves is the notion of convex hull, which highlights the issue that some points in the ROC curve can never be chosen as optimal points, since there are at least two other points in the curve for which an interpolation leads to a better point. This convexification of the ROC curve accounts for the idea that hybrid classifiers can also be constructed by interpolating between points which are not at consecutive rate isometrics.

Drummond and Holte (2006) state that the "ROC concept of upper convex hull also has an exact counterpart for cost curves: the lower envelope". While a correspondence between these two constructs can be established, this is only part of the story. First, the correspondence assumes optimal thresholds, and it is important to stress that the convex hull by itself 
does not imply that thresholds will be chosen optimally (as the lower envelope does). Second, and as a consequence of this, the area under the lower envelope is not even monotonically related to the area under the convex hull of the ROC curve. Clearly, the lower envelope of cost lines cannot be considered the "exact counterpart" of the ROC convex hull. The discovery of the rate-driven curve, which relates ROC space and cost space in a pointwise manner, suggests that the exact counterpoint of the ROC convex hull in cost space does indeed exist.

Definition 4 The convex skull of a rate-driven curve of a model $m$ is defined as the ratedriven curve of the convexified model $\operatorname{Conv}(m)$ (its convex hull in ROC space). The convex skull of a Kendall curve of a model $m$ is defined as the Kendall curve of $\operatorname{Conv}(m)$.

An example is shown in Fig. 5. The top solid line is the counterpart of the ROC curve while the top dashed line is the counterpart of the convex hull. We call this counterpart the convex skull, since it is geometrically related to the notion of convex skull, which is the biggest convex polygon which fits inside a non-convex polygon (Chang and Yap 1986). The difference in our case is that we do not really have polygons, since the segments are parabolic. Fortunately, there is no need to apply any complex algorithm to calculate the convex skull. There are two options for calculating $\operatorname{Conv}(m)$ in Definition 4 above.

One option is to calculate the convex hull geometrically in ROC space. A second option is to apply the Pair Adjacent Violators (PAV) algorithm (Fawcett and Niculescu-Mizil 2007) directly to the ranking. Given a set of training cases ordered by the scores assigned by a classification model, the PAV algorithm first assigns a probability 0 to each positive instance and a probability 1 to each negative instance creating a group for each instance. The algorithm then looks, at each iteration, for "adjacent violators": adjacent groups whose probabilities locally decrease rather than increase. In these cases, the algorithm pools the groups and replaces their probability estimates with the average of the group's values. This process stops when the entire sequence is monotonically non-decreasing. The result is a sequence of instances, each of which has a score and an associated probability estimate, which can then be used to map scores into probability estimates and recalculate the rank (with ties). The equivalence of these two ways of calculating Conv has been recently shown in Fawcett and Niculescu-Mizil (2007), where the algorithm is directly linked to the convex hull.

In fact, this second option is very easy to apply if we work with the Kendall curve (bottom solid line in Fig. 5). As we discussed in previous sections, horizontal segments correspond to positives or negatives (depending on which side of the rate isometric $\pi_{0}$ we are). This convex hull of the Kendall curve (which is lower convex for two portions, one from cost 0 to $\pi_{0}$ and the other one from cost $\pi_{0}$ to 1 ) is shown in the bottom dashed line in Fig. 5.

The first clear outcome is that the area under the convex skull follows the same linear relation to the convex hull in ROC space as established by Theorem 1 . The second outcome is that we can now better understand what the lower envelope means and its relation to the convex hull. Specifically, the lower envelope is an optimal cost curve, showing the loss for optimal decisions. This is an idealistic situation, since it assumes that the optimal thresholds in the training or validation data set for each operating condition will be valid as well for any future test set. The convex skull, on the other hand, shows the loss for the rate-driven threshold choice method after applying the PAV algorithm to the ranking. It gives a new interpretation of the convex hull in ROC space as a measure of classification performance of a model which has been processed by the PAV algorithm.

In the example in Fig. 5, the convex skull and the lower envelope only match for $c=0.2$. For this cost proportion, the rate leads to split the ranking: 0010001010 after the two 
Fig. 5 The solid line on top is the rate-drive curve with the dashed line just below its convex skull. The bottom pair of solid and dashed lines are the Kendall curve and its lower convex hull. The dashed line in the middle is the lower envelope of the cost lines

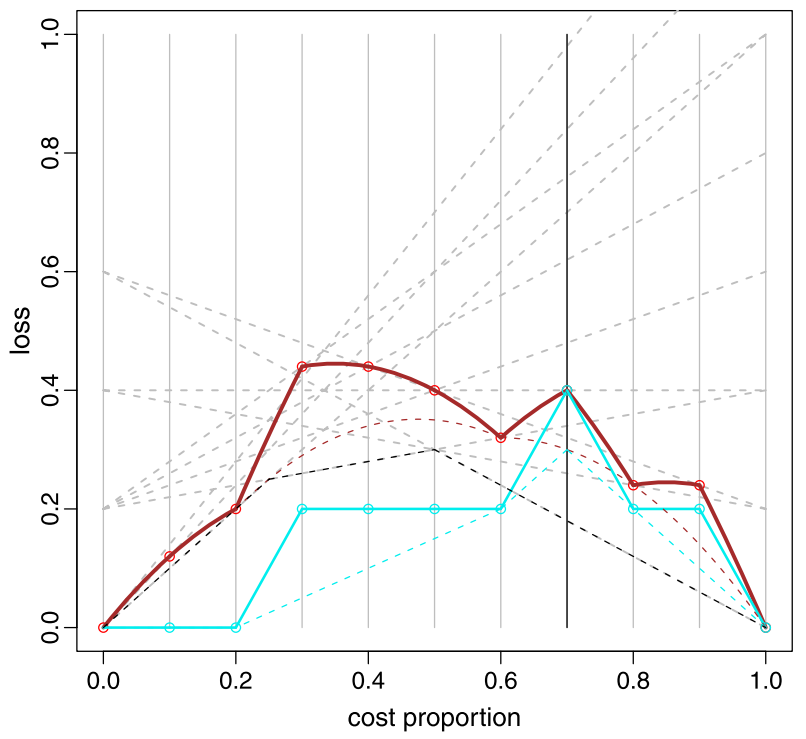

first 0 s. This gives the lowest loss for $c=0.2$ for this ranking ( 5 zeros misclassified as ones, with cost 0.2 each makes a total loss of 1.0 , which cannot be improved at any other split). We can see that the lower envelope can be attained by shifting the points of the convex skull along their corresponding cost line, to the right or to the left depending on their position. This shows that the convex skull does not represent optimal choices with respect to the operating condition.

\section{Partial areas and illustrative examples}

Screening is one of the most common applications in data mining. The goal of screening is to rank the instances in terms of the probability of an event (e.g. purchase, failure, disease, etc.) in order to find the greatest percentage of positive cases with the minimum percentage (or rate) of data inspected. Typical examples of screening applications are offer/mailing campaign design (e.g., in e-commerce, customer relationship management, etc.) or prevention policies (e.g., in medicine). Since ranking quality is crucial for this task, one common evaluation metric for the evaluation of ranking classifiers in these applications is the AUC.

However, it is almost never the case that we are interested in the performance of a model from an inspection rate of $0 \%$ to an inspection rate of $100 \%$. Typically, we work with some economic constraints about the minimum and maximum rates that are sensible in the application domain. In other words, we may be interested in the partial performance in a range of inspection rates.

Let us consider again the running example we introduced in Fig. 1, which had the ranking: 0010001010 over a training or validation set. Let us call it model $A$. Its $A U C$ was $13 / 21$. This ranking is neatly represented by the Kendall curve (bottom solid line) in Fig. 5. Now consider another model $(B)$ with the following ranking: 000010101000 over the same data set. The ranking is represented by the ROC curve and Kendall curve in Fig. 7. The $A U C$ is $11 / 21$. While the overall quality of model $B$ is worse than model $A$, both ROC curves cross at some points, so we cannot say that one model dominates the other for 

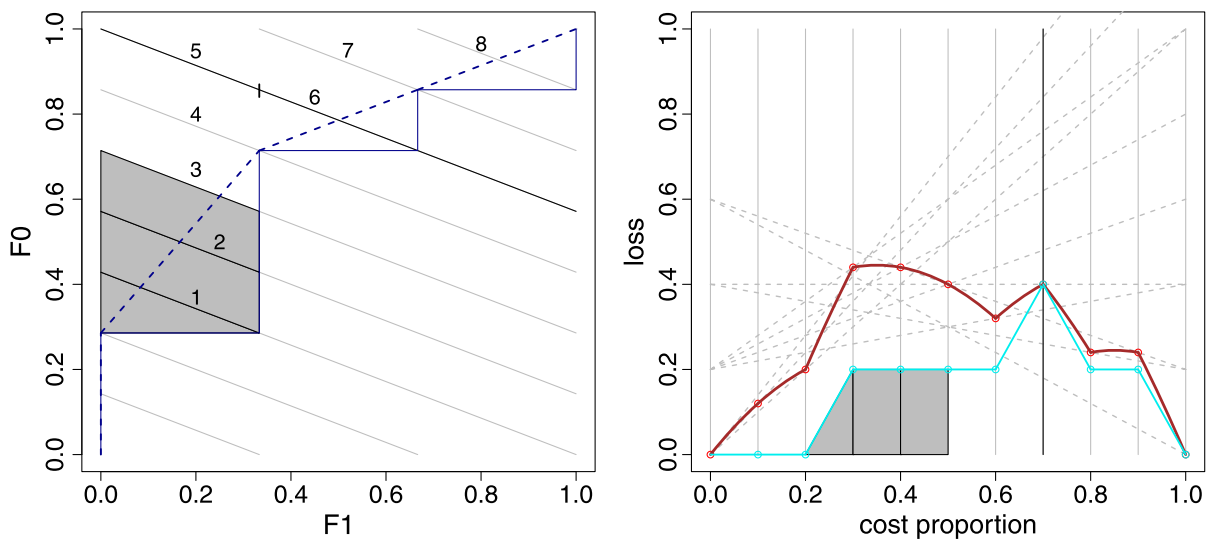

Fig. 6 Model A. ROC curve (left) and Kendall curve (right). The shadowed polygons represent partial areas over the ROC curve (left) and under the Kendall curve between rates 0.1 and 0.5 . This area is $(0.1+0.2+0.2) / 10=0.05$. The numbers on the ROC curve (left) from 1 to 8 represent the diagonals $D_{0}$ and $D_{1}$ as for Proposition 1
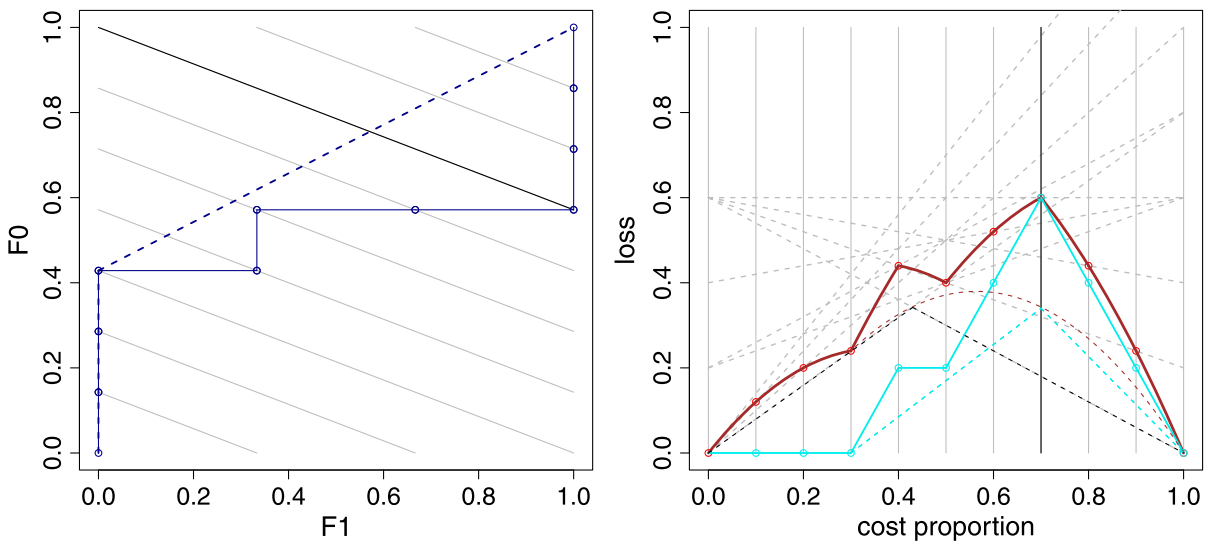

Fig. 7 Model B. Left: ROC curve for the ranking 0001011000 . Right: Kendall curve with its convex skull at the bottom, and rate-driven cost curve with its convex skull at the top. The remaining dashed line is the optimal cost curve

the whole range of operating conditions. However, current practice in screening applications would just simply choose model $A$ (if hybridisation between both models is not possible).

Imagine that we want to use this model to plan a mailing campaign for a new set of customers. The existence of constant costs for the mailing campaign suggests that the offer should be sent to no less than $10 \%$ of the customers. Also, according to budget limitations, the number of offers cannot exceed $50 \%$. These two constraints imply that we are interested in the quality of the ranking between $10 \%$ and $50 \%$ predicted positives. Which model is best in this situation? The problem of this question is that we are not asking what model is best in a specific operating condition (e.g., a rate equal to $40 \%$ ), but in a range of operating conditions. Interestingly, Kendall curves are the right plot to answer this question, because we can calculate partial areas in a straightforward way. In Fig. 6, we can see that the partial area under the Kendall curve between rates 0.1 and 0.5 is exactly 0.05 for model $A((0.2 / 2+$ 
$0.2+0.2) / 10)$. However, in Fig. 7 we see that this partial area for model $B$ is just 0.03 $((0.2 / 2+0.2) / 10)$. Consequently, for this range of contexts, model $B$ is preferable over model $A$.

It can be argued that these values can be calculated analytically. Of course they can, but it is much easier to see this in a plot with the Kendall curves, especially when we have thousands of examples (and not ten such as here). We can see the regions where each model dominates, and we can quantify the ranking loss for every possible region. In addition, the convex skull gives us information about the cutpoints that are sub-optimal. For instance, for model $A$ (Fig. 5), we know that in the range of rates between 0.1 and 0.5 , we should never choose $0.1,0.3$ and 0.4 , because the ranking is 0010001010 , and one can get more positives ( 0 ) further right on the ranking (e.g., 0.1 makes just the first example a true positive, while 0.2 makes the two first examples true positives). Note that this is just seen as horizontal segments in the Kendall curves. Similarly, for model $B$ (Fig. 7), we should never choose $0.1,0.2$ and 0.4 .

This information can also be obtained in the ROC space, especially with the equivalence we derived in Theorem 2. This way we can calculate that the partial AOC (between rates 0.1 and 0.5 ) for model $A$ is 0.119 while it is 0.071 for model $B$. We can even show this area between two isometrics in the ROC curves. However, this procedure is certainly much more difficult than in the cost space.

This application of Kendall curves is related to their interpretation in terms of the screening applications we are considering now: the area under the Kendall curve between two rates $r_{1}$ and $r_{2}$ represents how many screening mistakes one would make on average if all the cutpoints between $r_{1}$ and $r_{2}$ were considered equiprobable. This is the same approach as in Flach et al. (2011), but now we show it graphically and for partial regions. In fact, if one has further information about the distribution of the rates (e.g., if one thinks that a $20 \%$ for an inspection rate is more likely than $30 \%$ ), then we could just 'warp' the $x$-axis of the plots using this information (as a distribution) and calculate the area accordingly.

While we have illustrated this for ranking models, this can also be shown for classification models using the rate-driven threshold choice method. For instance, if we have a spam filtering model, we can have information (or make the assumption) that a false positive (predicted spam being actual ham) will always have higher cost than a false negative (predicted ham being actual spam), i.e. $c_{1}>c_{0}$ (and clearly $c<0.5$ ). This means that we could compare models by looking at their partial rate-driven cost curves. In this case, we would just calculate the area under the rate-driven cost curve between rates 0 and $\pi_{0}$. This would tell us which model is best for that range of operating conditions using the rate-driven threshold choice method.

We can see all this in practice for a more realistic example. We chose the German credit data set (Frank and Asuncion 2010) because it is illustrative for screening applications and cost-sensitive problems. Class 0 represents good customers (profitable customers for a credit) while class 1 represents bad customers. Figure 8 compares two models (a $k$-nearest neighbours, $k \mathrm{NN}$, mostly top, and a decision tree, J48, mostly bottom) using cost space and their rate-driven cost curves.

If we want to use these two models for classification using the rate-driven threshold choice method, we can see that, depending on the operating condition, one model can be better than the other. Typically, bad customers classified as good customers (false positives) have much higher cost than false negatives (the German credit data set sets this ratio to 5:1). Since the prior distribution may vary and the particular cost matrix may depend on other circumstances, it is reasonable to analyse both models in a range of operating conditions. Let us assume that we are given a region of, say, rates between 0 and 0.35 . With this region, 
Fig. 8 Two Kendall curves for the German credit data set using $k$-nearest neighbours (mostly top) and a decision tree (mostly bottom). The solid vertical line shows $\pi_{0}=0.69$. The vertical dotted lines show the region of interest

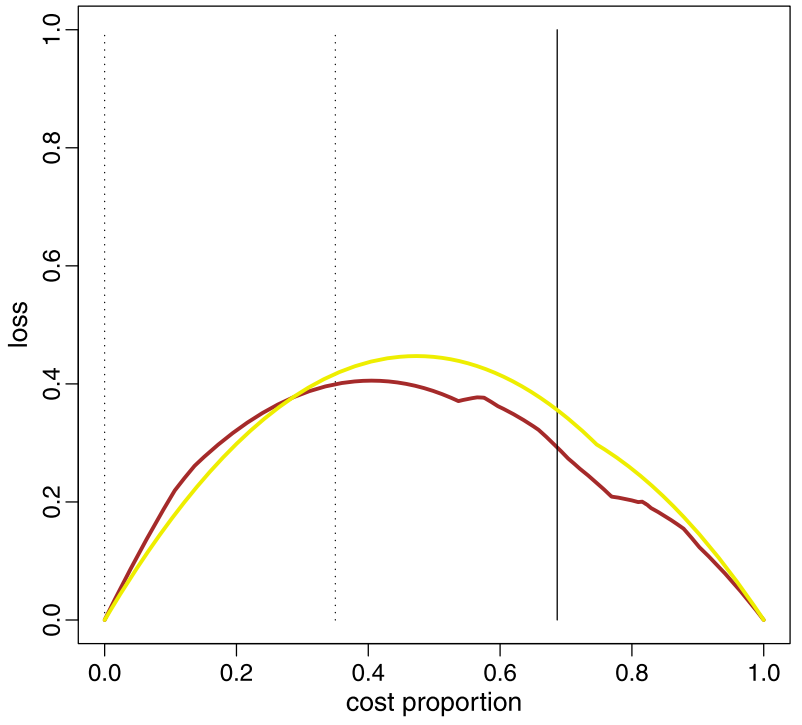

which is shown with dotted vertical lines in Fig. 8, we can calculate the partial areas of the rate-driven cost curves (between 0 and 0.35), which are 0.093 for the J48 model, and 0.091 for the $k \mathrm{NN}$ model. Consequently, the $k \mathrm{NN}$ model is better for the range of rates we want to consider. This contrasts with the total area, which, in this case, is lower (better) for the J48 model (0.27) than the $k \mathrm{NN}$ model (0.29).

Interestingly, the same choice would be obtained for any partial calculation using the rate-driven cost curve or the Kendall curve. However, if we want to calculate the expected misclassification loss, then it is the rate-driven cost curve we need to look at. If we want to calculate the expected number of misclassifications for a screening application, then it is the Kendall curve we would look at.

\section{Concluding remarks}

The definition of cost curve in the literature has been partially elusive. While it is clear what cost lines are, it was not clear what the options are for drawing different curves in cost space, which of them were valid and, more importantly, whether they correspond to curves or representations in ROC space. In this paper we have clarified the relation between both spaces, by defining the rate-driven cost curve as the true companion of ROC curves in cost space. We have furthermore demonstrated that it is possible to visualise classification performance and ranking performance in the same plot by means of the Kendall curve.

Our main instrument was the rate-driven threshold choice method, which leads to a pointpoint correspondence between the ROC curve and the rate-driven curve, and also between the ROC convex hull and the convex skull. This provides a richer view of cost space, since different cost curves arising from different threshold choice methods can be contrasted and compared.

While cost curves were initially introduced for skews, we have worked with cost proportions in this paper, but a generalisation to skews should be straightforward. We plan to work on the use of rate-driven curves to choose among models and construct hybrid classifiers. 
Another interesting avenue for further work is a comparison with the recently proposed Brier curves (Hernández-Orallo et al. 2011), especially because it has been shown in Hernández-Orallo et al. (2012) that the rate-driven threshold choice method is equal to the score-driven threshold choice method when scores are evenly spaced (however, Brier curves do not interpolate and their exact equivalence in this particular score disposition would only be asymptotical). By comparing different curves in the same space we should be able to decide which threshold choice method is best for a particular operating condition, leading to a new dimension of dominance. This comparison of several curves (using different threshold choice methods) would usually be carried out for different data sets. For instance, we could plot the curves using a labelled training data set, from which the threshold choices could be derived for each operating condition, and then these choices could be used to represent curves on a different labelled validation data set. This would show that some curves may be too optimistic on the training data set and may lead to worse choices on the validation data set.

The source code in $\mathrm{R}$ for plotting rate-driven curve sand Kendall curves can be found at http://users.dsic.upv.es/ flip/RDC/.

Acknowledgements We would like to thank the anonymous referees for their helpful comments. This work was supported by the MEC/MINECO projects CONSOLIDER-INGENIO CSD2007-00022 and TIN 2010-21062-C02-02, GVA project PROMETEO/2008/051, the COST-European Cooperation in the field of Scientific and Technical Research IC0801 AT, and the REFRAME project granted by the European Coordinated Research on Long-term Challenges in Information and Communication Sciences \& Technologies ERA-Net (CHIST-ERA), and funded by the Engineering and Physical Sciences Research Council in the UK and the Ministerio de Economía y Competitividad in Spain.

\section{References}

Adams, N., \& Hand, D. (1999). Comparing classifiers when the misallocation costs are uncertain. Pattern Recognition, 32(7), 1139-1147.

Chang, J., \& Yap, C. (1986). A polynomial solution for the potato-peeling problem. Discrete \& Computational Geometry, 1(1), 155-182.

Drummond, C., \& Holte, R. (2000). Explicitly representing expected cost: an alternative to ROC representation. In Knowl. discovery \& data mining (pp. 198-207).

Drummond, C., \& Holte, R. (2006). Cost curves: an improved method for visualizing classifier performance. Machine Learning, 65, 95-130.

Elkan, C. (2001). The foundations of cost-sensitive learning. In B. Nebel (Ed.), Proc. of the 17th intl. conf. on artificial intelligence (IJCAI-01) (pp. 973-978).

Fawcett, T. (2006). An introduction to ROC analysis. Pattern Recognition Letters, 27(8), 861-874.

Fawcett, T., \& Niculescu-Mizil, A. (2007). PAV and the ROC convex hull. Machine Learning, 68(1), 97-106.

Flach, P. (2003). The geometry of ROC space: understanding machine learning metrics through ROC isometrics. In Machine learning, proceedings of the twentieth international conference (ICML 2003) (pp. 194-201).

Flach, P., Hernández-Orallo, J., \& Ferri, C. (2011). A coherent interpretation of AUC as a measure of aggregated classification performance. In Proc. of the 28th intl. conference on machine learning, ICML2011.

Frank, A., \& Asuncion, A. (2010). UCI machine learning repository. http://archive.ics.uci.edu/ml.

Hand, D. (2009). Measuring classifier performance: a coherent alternative to the area under the ROC curve. Machine Learning, 77(1), 103-123.

Hernández-Orallo, J., Flach, P., \& Ferri, C. (2011). Brier curves: a new cost-based visualisation of classifier performance. In Proceedings of the 28th international conference on machine learning, ICML2011.

Hernández-Orallo, J., Flach, P., \& Ferri, C. (2012). A unified view of performance metrics: translating threshold choice into expected classification loss. Journal of Machine Learning Research, 13, 2813-2869.

Kendall, M. G. (1938). A new measure of rank correlation. Biometrika, 30(1/2), 81-93. doi:10.2307/ 2332226.

Swets, J., Dawes, R., \& Monahan, J. (2000). Better decisions through science. Scientific American, 283(4), $82-87$. 